\title{
An Online Outlier Detection Technique for Wireless Sensor Networks using Unsupervised Quarter-Sphere Support Vector Machine
}

\author{
Yang Zhang \\ Department of Computer Science \\ University of Twente \\ Enschede, The Netherlands \\ Email: zhangy@cs.utwente.nl
}

\author{
Nirvana Meratnia \\ Department of Computer Science \\ University of Twente \\ Enschede, The Netherlands \\ Email: N.Meratnia@ewi.utwente.nl
}

\author{
Paul Havinga \\ Department of Computer Science \\ University of Twente \\ Enschede, The Netherlands \\ Email: P.J.M.Havinga@ewi.utwente.nl
}

\begin{abstract}
The main challenge faced by outlier detection techniques designed for wireless sensor networks is achieving high detection rate and low false alarm rate while maintaining the resource consumption in the network to a minimum. In this paper, we propose an online outlier detection technique with low computational complexity and memory usage based on an unsupervised centered quarter-sphere support vector machine for real-time environmental monitoring applications of wireless sensor networks. The proposed approach is completely local and thus saves communication overhead and scales well with increase of nodes deployed. We take advantage of spatial correlations that exist in sensor data of adjacent nodes to reduce the false alarm rate in real-time. Experiments with both synthetic and real data collected from the Intel Berkeley Research Laboratory show that our technique achieves better mining performance in terms of parameter selection using different kernel functions compared to an earlier offline outlier detection technique designed for wireless sensor networks.
\end{abstract}

\section{INTRODUCTION}

Wireless sensor networks (WSNs) have been widely used in various applications including those related to personal, industrial, business and military domains [1]. Many of these applications utilize real-time sensor data collected by WSNs to monitor the surrounding environment and detect time-critical events occurred in the physical world.

Data collected by WSNs are often unreliable and inaccurate due to the following reasons: (i) the low cost and low quality sensor nodes have stringent resource constraints such as energy (battery power), memory, computational capacity, and communication bandwidth; (ii) operation of sensor nodes which are randomly deployed in a large area (and often with high density) are frequently susceptible to harsh and unattended environmental effects; (iii) sensor nodes are vulnerable to malicious attacks such as denial of service attacks, black hole attacks and eavesdropping [2, 3]. To keep the data quality and reliability high and be able to make effective and correct decisions using data collected by WSNs, it is essential to identify erroneous data as well as potential events and malicious attacks occurred in the network. Outliers in WSNs are those measurements that significantly deviate from the normal pattern of the sensed data [4].
The context of sensor networks and nature of sensor data make design of an appropriate outlier detection technique challenging. The main challenge faced by outlier detection techniques designed for WSNs is achieving high detection rate and low false alarm rate while maintaining the resource consumption of WSNs to a minimum. In other words, outliers in WSNs should be detected locally in the network and in real-time with a low communication overhead, memory and computation cost.

In this paper, we propose an online and local outlier detection technique with low resource consumption based on an unsupervised (one-class) centered quarter-sphere support vector machine (SVM) for environmental monitoring applications of WSNs. This approach takes advantage of spatial correlations that exist in sensor data of adjacent nodes to reduce the false alarm rate and to distinguish between events and errors in real-time. Experiments with synthetic and real data of Intel Berkeley Research Laboratory [10] show that our approach achieves better mining performance in terms of parameter selection with different kernel functions compared to an earlier offline outlier detection technique for WSNs mentioned in [5].

The rest of this paper is organized as follows. Related work on one-class SVM-based outlier detection techniques is presented in Section II. The problem statement and fundamentals of the one-class centered quarter-sphere SVM are described in Section III. Our proposed online and local outlier detection technique is explained in Section IV. Experimental results and performance evaluation of our approach are reported in Section V. We conclude the paper in Section VI with plans for future research.

\section{RELATED WORK}

Outlier detection has been widely researched in various disciplines such as statistics, data mining, machine learning, information theory, and spectral decomposition [4]. Outlier detection techniques designed for WSNs can be categorized into statistical-based, nearest neighbor-based, clusteringbased, classification-based, and spectral decomposition-based approaches [6]. Classification-based approaches are important 
systematic approaches in the data mining and machine learning community. Classification-based techniques learn a classification model using a set of data instances in the training phase and classify an unseen instance into one of the learned (normal/outlier) class in the testing phase [4]. SVM-based techniques are from family of classification-based approaches and have the following three main advantages:

- have a simple geometric interpretation;

- provide an optimum solution for classification by maximizing the margin of the decision boundary;

- avoid the problem of the curse of dimensionality.

The fact that in many WSNs applications, pre-classified normal/anomalous data is neither always available nor easy to obtain implies that unsupervised classification techniques suit the WSNs the best. Therefore, several unsupervised (one-class) SVM-based outlier detection techniques have been proposed $[7,8,9,5]$, which model the normal pattern of the unlabelled data while automatically ignore the anomalies existed in the training data. The main idea of one-class SVM-based outlier detection approaches is that data measurements collected from the original space (input space) are first mapped to a higher dimensional space (feature space) using a non-linear function $\phi(x)$. Then a decision boundary of normal data is found, which encompasses the majority of the data measurements in the feature space. Those falling outside the boundary are classified as anomalous. Scholkopf et al. [7] have proposed a hyperplanebased one-class SVM for outlier detection. Tax et al. [8] have proposed a hypersphere one-class SVM for outlier detection, which has a more intuitive geometric idea. The idea is that a hypersphere is fitted with a minimal radius to encompass the majority of data in the feature space. Those falling outside the hypersphere indicate anomalous data.

One of the problems of one-class SVM-based outlier detection techniques using the hyperplane and the hypersphere is that they involve a quadratic optimization process during learning the boundary of normal data. This process is extremely costly and not suitable for limited resources available in WSNs. Laskov et al. [9] have extended work in [8] by proposing a one-class quarter-sphere SVM, which is formulated as a linear optimization problem and thus reduces the effort and computational complexity. Rajasegarar et al. [5] further exploit potential of the one-class quarter-sphere SVM of [9] for outlier detection in WSNs. However, their technique detects outliers only after data measurements are being collected for a long period of time and thus it is not suitable for real-time environmental monitoring applications of WSNs. This offline technique is also costly in terms of memory usage. Moreover, this approach identifies local outliers only in a node depending on the SVM classifier learned by itself, which may result in high false alarm rate due to the lack of sufficient information.

In this paper, we extend the offline approach of [5], and propose an online and local outlier detection technique with low resource consumption. By taking advantage of spatial correlations that exist in sensor data of adjacent nodes we show that false alarm rate can be reduced and even real-time distinction between events and errors can be made.

\section{Fundamentals OF ONE-Class CENTERED QUARTER-SPHERE SVM}

\section{A. Problem Statement}

We consider that sensor nodes are time synchronized and are densely deployed in a homogeneous WSN, where sensor data tends to be correlated in both time and space. The network topology is modelled as an undirected graph $G$ where $G=(S$, $E)$. $S$ represents the nodes in the network and $E$ represents an edge which connects two nodes if they are within radio transmission range of each other. A subset $N\left(S_{i}\right)$ represents a closed neighborhood of a node $S i \epsilon S$, which contains the node $S_{i}$ and its $k$ spatially neighboring nodes. The $k$ spatially neighboring nodes are represented by $S_{i j}=\left\{S_{i j}: j=1 \ldots k\right\}$, i.e., $N\left(S_{i}\right)=\left\{S_{i j} \epsilon S \mid\left(S_{i j}, S_{i}\right) \in E\right\} \cup\left\{S_{i}\right\}$. An example of $N\left(S_{i}\right)$ is the closed disk centered at $S_{i}$ with the radio transmission range of $S_{i}$, as shown in Figure (1).

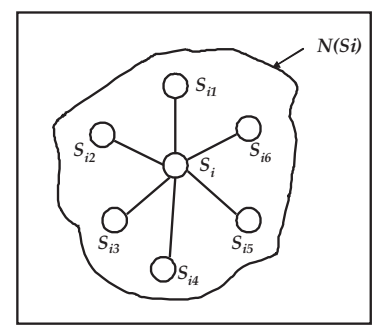

Fig. 1. Example of a closed neighborhood $N\left(S_{i}\right)$ of the sensor node $S_{i}$

At every time interval $\Delta_{t}$, each sensor node in the set $N\left(S_{i}\right)$ measures a data vector. Let $x_{i}, x_{1}^{i}, x_{2}^{i}, \ldots, x_{k}^{i}$ denote the data vector measured at $S_{i}, S_{i 1}, S_{i 2}, \ldots, S_{i k}$, respectively. Each data vector is composed of multiple attributes $x_{j l}^{i}$, where $x_{j}^{i}=\left\{x_{j l}^{i}: j=0 \ldots k, l=1 \ldots d\right\}$ and $x_{j}^{i} \in \Re^{d}$. Our aim is to identify every new data measurement arriving at $S_{i}$ as normal or anomalous in real-time. This local process can be applied to each node in the network and thus scales well to large WSNs.

\section{B. One-Class Centered Quarter-Sphere SVM}

By fixing the center of the quarter-sphere at the origin, Laskov et al. [9] have converted the quadratic optimization problem of one-class SVM-based to a linear optimization problem. The geometries of the two approaches are shown in Figure (2).

The constrained optimization problem of the one-class centered quarter-sphere SVM is formalized as follows:

$$
\min _{R \in \Re, \xi \epsilon \Re^{m}} R^{2}+\frac{1}{v m} \sum_{i=1}^{m} \xi_{i}
$$

subject to: $\left\|\phi\left(x_{i}\right)\right\|^{2} \leq R^{2}+\xi_{i}, \quad \xi_{i} \geq 0, \quad i=1,2, \ldots m$

where $\left\{\xi_{i}: i=1,2, \ldots m\right\}$ are the slack variables that allow some of the data vectors to fall outside the quartersphere. The number of data vectors is denoted by $m$. The 


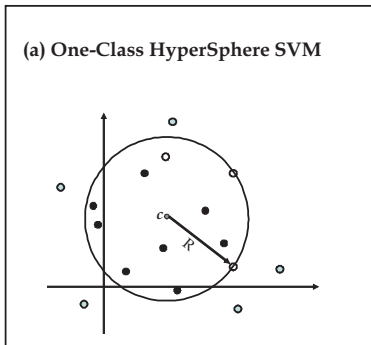

(b) One-Class Centered Quarter-Sphere SVM

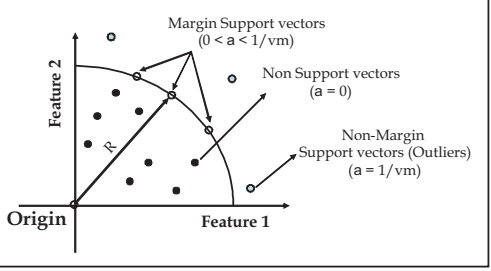

Fig. 2. (a) Geometry of the hypersphere formulation of one-class SVM. (b) Geometry of the quarter-sphere formulation of one-class SVM for $c=0$

parameter $v \in(0,1)$ is the regularization parameter that represents the fraction of anomalous data vectors (outliers). The Lagrange function for this optimization is:

$L=R^{2}-\sum_{i=1}^{m} \alpha_{i}\left(R^{2}-\left\|\phi\left(x_{i}\right)\right\|^{2}+\xi_{i}\right)-\sum_{i=1}^{m} \beta_{i} \xi_{i}+\frac{1}{v m} \sum_{i=1}^{m} \xi_{i}$

where $\alpha_{i} \geq 0, \beta_{i} \geq 0$ for all $i=1,2, \ldots, m$ are the Lagrangian multipliers. Taking the derivative of $L$ with respect to $R$ and $\xi_{i}$ to zero result to:

$$
\begin{aligned}
& \frac{\partial L}{\partial R}=0 \quad \Rightarrow \quad \sum_{i=1}^{m} \alpha_{i}=1 \\
& \frac{\partial L}{\partial \xi_{i}}=0 \quad \Rightarrow \quad \alpha_{i}=\frac{1}{v m}-\beta_{i}
\end{aligned}
$$

From (4), we can obtain $0 \leq \alpha_{i} \leq \frac{1}{v m}$ using $\alpha_{i} \geq 0$, $\beta_{i} \geq 0$. Substituting (3) and (4) into (2) produces:

$$
L=\sum_{i=1}^{m} \alpha_{i}\left(\phi\left(x_{i}\right) \cdot \phi\left(x_{i}\right)\right)
$$

where the dot product $\phi\left(x_{i}\right) \cdot \phi\left(x_{i}\right)$ is regarded as a measure of similarity between $\phi\left(x_{i}\right)$ and $\phi\left(x_{i}\right)$ in the feature space. It can be replaced by a kernel function $k\left(x_{i}, x_{i}\right)$, which is a method for computing similarity in the feature space using the original attribute set [13]. Hence, the dual formulation of (1) will become:

$$
\min _{\alpha \in \Re^{m}}-\sum_{i=1}^{m} \alpha_{i} k\left(x_{i}, x_{i}\right)
$$

$$
\text { subject to }: \sum_{i=1}^{m} \alpha_{i}=1, \quad 0 \leq \alpha_{i} \leq \frac{1}{v m}, \quad i=1,2, \ldots m
$$

Converting the dual problem of a quadratic optimization to a linear optimization problem effectively reduces the computational complexity. In order to fix the center of the quartersphere at the origin, the mapped data vectors in the feature space need to be subtracted from the mean. The mean is represented as:

$$
\mu=\frac{1}{m} \sum_{i=1}^{m} \phi\left(x_{i}\right), \text { i.e., } \phi\left(x_{i}\right)_{c}=\phi\left(x_{i}\right)-\frac{1}{m} \sum_{i=1}^{m} \phi\left(x_{i}\right) .
$$

The centered kernel matrix $K_{c}$ can be obtained in terms of the kernel matrix $K=k\left(x_{i}, x_{j}\right)=\left(\phi\left(x_{i}\right) \cdot \phi\left(x_{j}\right)\right)$ using $K_{c}=K-1_{m} K-K 1_{m}+1_{m} K 1_{m}$, where $1_{m}$ is an $m \times m$ matrix with all values equal to $\frac{1}{m}$.

From equation (6), the $\left\{\alpha_{i}\right\}$ value can be easily obtained using some effective linear optimization techniques [14]. The data vectors can further be classified depending on the results of $\left\{\alpha_{i}\right\}$, as shown in Figure (2b). The data vectors with $\alpha=0$, which fall inside the quarter-sphere and their distances from the origin are smaller than the radius of the quarter-sphere, are called non support vectors. The data vectors with $\alpha>0$, which determine the computational complexity and accuracy of the learned SVM classifier, are called support vectors. Support vectors with $0 \leq \alpha \leq \frac{1}{v m}$, which fall on the quartersphere, are called margin support vectors. Their distances to the origin indicate the minimal radius of the quarter-sphere. Support vectors with $\alpha=\frac{1}{v m}$, which fall outside the quartersphere and their distances from the origin are larger than the radius of the quarter-sphere, are called non-margin support vectors. These are in fact the outliers that we are interested in identifying them.

\section{An Online AND Local OUtlier Detection TECHNIQUE FOR WIRELESS SENSOR NETWORKS}

Our online technique enables each sensor node in the network to identify its new arriving data measurements as normal or anomalous in real-time. Using the high degree of spatial correlations that exist among the sensor readings of the adjacent nodes in a densely deployed WSN, each node has sufficient information to detect local outliers. This detection does not only depend on a node's own decision criterion but also on the decision criteria learned from its spatially neighboring nodes. Identification of outlier type, i.e., making distinction between events and errors, can be made based on the observation that erroneous measurements are likely to be spatially unrelated, while event measurements are likely to be spatially correlated [12].

The pseudocode of our proposed outlier detection technique is shown in Table (I). Initially, each node learns the local radius of the quarter-sphere using its $m$ sequential data measurements, which may include some anomalous data. The one-class quarter-sphere SVM can efficiently find a minimal radius to enclose the majority of these mapped data measurements in the feature space. Each node then locally broadcasts the learned radius information to its spatially neighboring nodes. In fact a node $S_{i}$ first collects the radius information from all of its neighbors and then computes a median radius $R_{i m}^{\prime}$ of its neighboring nodes as well as a median radius $R_{i m}$ of its closed neighborhood $N\left(S_{i}\right)$. One should note that to estimate the "center" of a sample set, the median is more robust than the mean. 
When a new data measurement $x$ arrives at node $S_{i}$, it computes the distance $d(x)_{c}$ between $x$ and the origin of its centered quarter-sphere in the feature space. According to the mean $\mu=\frac{1}{m} \sum_{i=1}^{m} \phi\left(x_{i}\right)$ and the kernel matrix $K=$ $k\left(x_{i}, x_{j}\right)=\left(\phi\left(x_{i}\right) \cdot \phi\left(x_{j}\right)\right)$, the distance of $x$ from the origin in the feature space is formalized as follows:

$$
\begin{aligned}
d(x)_{c} & =\sqrt{\left\|\phi(x)-\frac{1}{m} \sum_{i=1}^{m} \phi\left(x_{i}\right)\right\|^{2}} \\
& =\sqrt{\|\phi(x)\|^{2}+\frac{1}{m^{2}} \sum_{i=1}^{m}\left\|\phi\left(x_{i}\right)\right\|^{2}-\frac{2}{m} \sum_{i=1}^{m} \phi(x) \cdot \phi\left(x_{i}\right)} \\
& =\sqrt{\phi(x) \cdot \phi(x)+\frac{1}{m^{2}} \sum_{i=1}^{m} \phi\left(x_{i}\right) \cdot \phi\left(x_{i}\right)-\frac{2}{m} \sum_{i=1}^{m} k\left(x, x_{i}\right)} \\
& =\sqrt{k(x, x)+\frac{1}{m^{2}} \sum_{i=1}^{m} k\left(x_{i}, x_{j}\right)-\frac{2}{m} k\left(x, x_{i}\right) .}
\end{aligned}
$$

Based on the fact that sensor data collected in a densely deployed WSN tends to be correlated in both time and space, $S_{i}$ first compares $d(x)_{c}$ obtained by (7) with its own radius $R_{i}$. The data $x$ will be classified as normal if $d(x)_{c}<=R_{i}$, which means that $x$ falls on or inside the quarter-sphere at $S_{i}$. Otherwise if $d(x)_{c}>R_{i}, x$ is a potential (temporal) outlier. In this case, $S_{i}$ further compares $d(x)_{c}$ with the median radius $R_{i m}^{\prime}$ of its spatially neighboring nodes. If $d(x)_{c}>R_{i m}^{\prime}, x$ will finally be classified as outlier in the set $N\left(S_{i}\right)$. Thus, the decision function can be formulated as:

$$
f(x)=\operatorname{sgn}\left(R_{i}-d(x)_{c}\right) \bigoplus \operatorname{sgn}\left(R_{i m}^{\prime}-d(x)_{c}\right)
$$

where the data measurements with $f(x)=-1$ are classified as outlier. $R_{i}$ and $R_{i m}^{\prime}$ are important decision criteria for local outlier identification.

Identifying what has caused the outlier in sensor data is an important task. Potential sources of outliers in data collected by WSNs include noise \& errors, actual events, and malicious attacks. Noisy data as well as erroneous data should be eliminated or corrected if possible as noise is a random error without any real significance that dramatically affects the data quality. Outliers caused by other sources need to be identified as they may contain important information about events that are of great interest to the researchers [6].

Our proposed technique provides a preliminary method to make real-time distinction between events and errors by using sensor data of neighboring nodes and their spatial similarity. The main idea is that only when a data measurement is considered as outlier, $S_{i}$ collects the distances of its neighboring nodes' currently sensing the data from their own origin in the feature space and computes a median distance $d_{i m}^{\prime}$. If an event occurs in the set $N\left(S_{i}\right), d(x)_{c}$ and $d_{i m}^{\prime}$ will be temporally different but a spatial consensus will be observed [12]. This means that $d(x)_{c}$ and $d_{i m}^{\prime}$ will exceed their own radius of $R_{i}$ and $R_{i m}^{\prime}$, respectively. In addition, they both will exceed the median radius $R_{i m}$ of the set $N\left(S_{i}\right)$. If this is not the case, the detected outlier may indicate an erroneous measurement.

TABLE I

THE PSEUDOCODE OF OUR PROPOSED OUTLIER DETECTION TECHNIQUE.

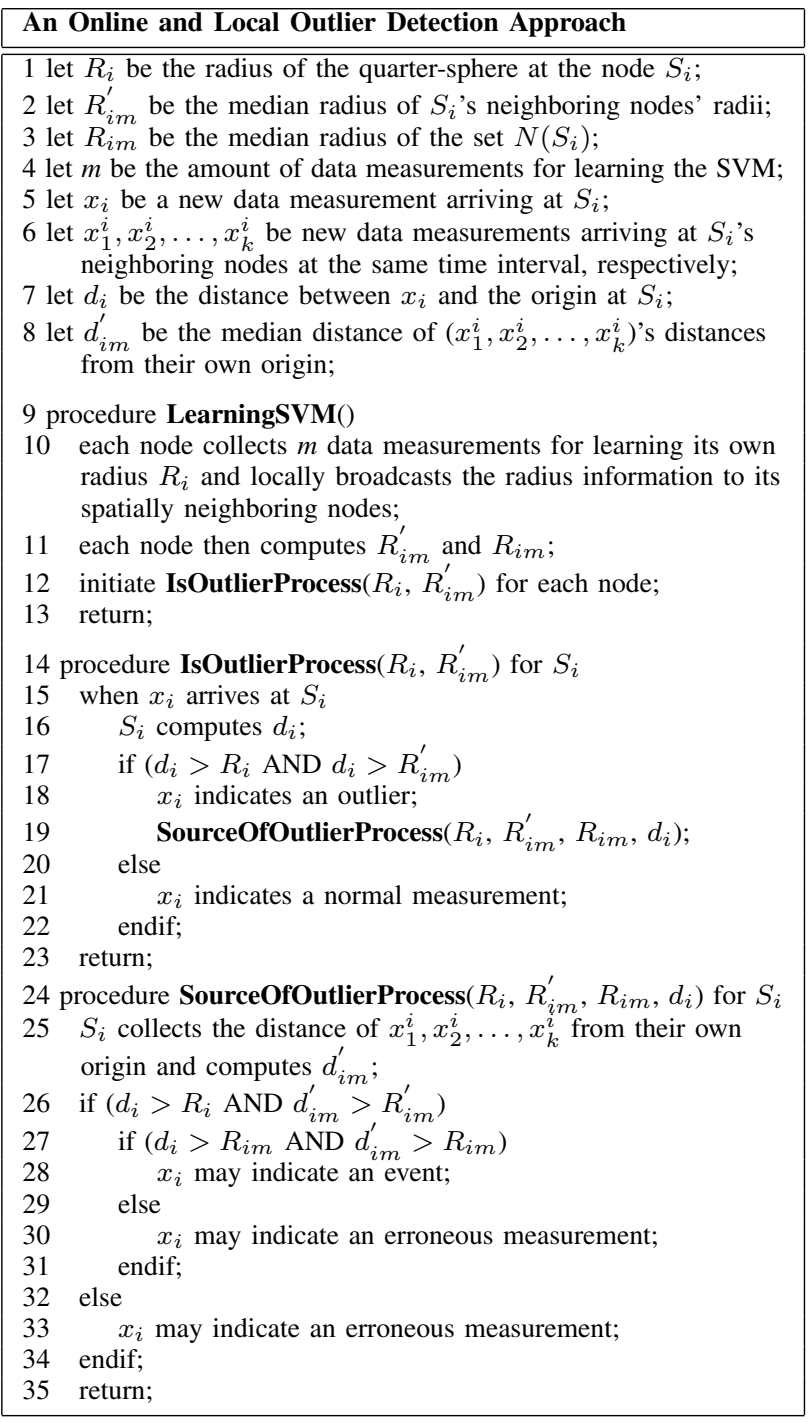

Our technique scales well with increase of number of nodes deployed in the network. The reason is that the local process is applied to each node in the network to enable the node to specify its new arriving data measurements as normal or anomalous in real-time. The computational complexity of our scheme is low as it only depends on solving a linear optimization problem. Once the optimization is solved, each node only keeps the radius value and the $m$ initial data measurements in memory. In contrary to the offline technique of [5], this prevents additional memory usage for saving new data measurements collected for a long period of time window. The use of spatial correlations provides each node with more 
sufficient information to correctly identify local outliers and even to distinguish between events and errors. Our scheme is suitable for outlier detection in real-time environmental monitoring applications of WSNs. The scheme also involves low communication overhead as it performs outlier detection at the node locally.

\section{EXPERIMENTAL RESUlts AND EVAluation}

This section specifies the performance evaluation of our technique compared to the earlier offline technique of [5]. In our experiments, we have used synthetic data as well as real data gathered from a deployment of WSN in the Intel Berkeley Research Laboratory [10, 11]. We simulate our protocol in Matlab and consider a closed neighborhood as shown in Figure (1), which is centered at a node with its 6 spatially neighboring nodes.

\section{A. Synthetic Dataset}

The 2-D synthetic data used for each node is composed of a mixture of three Gaussian distribution with uniform outliers; the mean is randomly selected from $(0.3,0.35,0.45)$, and the standard deviation is selected as 0.03 . Subsequently, $5 \%$ (of the normal data) anomalous data is introduced and uniformly distributed in the interval $[0.5,1]$. The data values are normalized to fit in the $[0,1]$. To have a fair comparison and due to the fact that the offline technique of [5] evaluates its performance using the original training data with added labels rather than new testing data, thus in the experiment we use the same amount of data measurements in both techniques for training the quarter-sphere SVM classifier while the same amount of new testing data is used to evaluate the performance of our technique. The testing data used for the centered node comprises of 200 normal and 10 anomalous data, which is plotted as shown in Figure (4a).

\section{B. Real Dataset}

The real data are collected from a closed neighborhood from a WSN deployed in the Intel Berkeley Research Laboratory as shown in Figure (3). The closed neighborhood contains the node 35 and its 6 spatially neighboring nodes, namely nodes 1 , $2,33,34,36,37$. The network recorded temperature, humidity, light and voltage measurements at 31 seconds intervals. In our experiments, we use a $9 \mathrm{am}-17 \mathrm{pm}$ period of data recorded on 5th March 2003 with two attributes: temperature and humidity for each data measurement. The data values are normalized to the range $[0,1]$. The amount of anomalous data is about $5 \%$ $10 \%$ of normal data. The labels of data measurements are obtained depending on the degree of dissimilarity between each other.

As shown in Figure (4b), the data vectors are labelled as anomalous if they are distant from other data vectors. The same amount of new testing data as the training data is used to evaluate the performance of our technique.

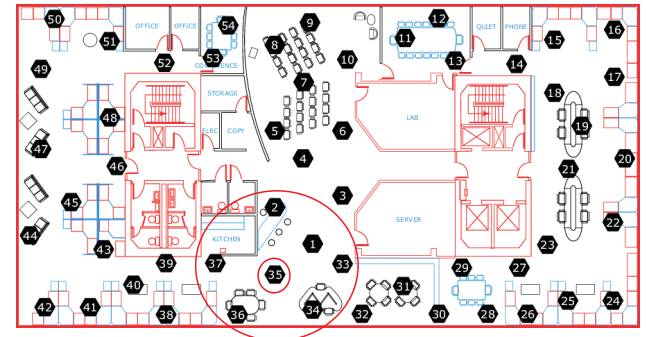

Fig. 3. Sensor nodes deployed in Intel Berkeley Research Laboratory [10]
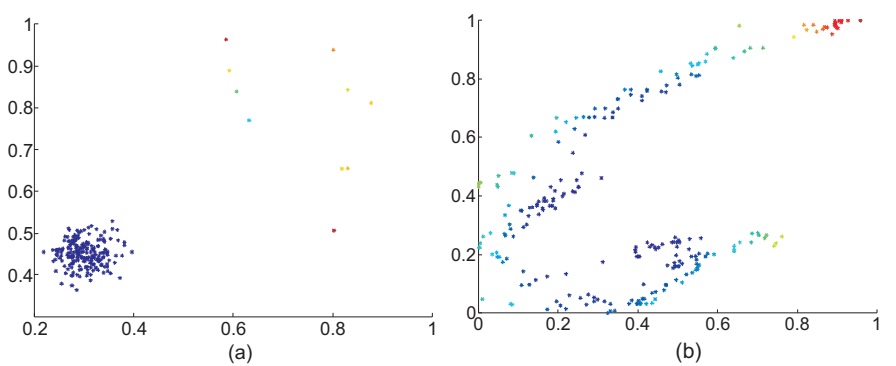

Fig. 4. (a) Plot for synthetic data. (b) Plot for real data.

\section{Experimental Results and Evaluation}

We have tested the following three kernel functions:

1) Linear kernel function: $k_{\text {Linear }}=\left(x_{1} \cdot x_{2}\right)$, where $\left\{x_{1}, x_{2}\right\}$ are the data vectors;

2) Radial basis function ( $R B F$ ) kernel function: $k_{R B F}=$ $\exp \left(-\left\|x_{1}-x_{2}\right\|^{2} / \sigma^{2}\right)$, where $\sigma$ is the width parameter of the kernel function;

3) Polynomial kernel function: $k_{\text {Polynomial }}=\left(x_{1} \cdot x_{2}+1\right)^{r}$, where $r$ is the degree of the polynomial.

Kernel matrices generated using the above kernel functions were centered. We have evaluated two important performance metrics, the detection rate, which represents the percentage of anomalous data that are correctly considered as outliers, and the false alarm rate, also known as false positive rate (FPR), which represents the percentage of normal data that are incorrectly considered as outliers.

We have examined the effect of the regularisation parameter $v$ for the two outlier detection techniques, i.e., our online proposed technique and offline approach presented in [5], using the linear, RBF and polynomial kernel functions. $v$ represents the fraction of outliers and in the experiments we have varied it in the range from 0.01 to 0.25 in intervals of 0.01 . A receiver operating characteristics (ROC) curves is usually used to represent the trade-off between the detection rate and the false alarm rate. However, since the simulation results show that both techniques achieve $100 \%$ detection rate in most of cases. Therefore, the ROC curve would not be a useful tool to compare the the performance of the two techniques. Thus, we show the simulation results with the false alarm rate in terms of parameter $(v)$ selection using different kernel functions. As a matter of fact, the main challenge of unsupervised techniques for outlier detection is how to reduce the high false alarm rate. 

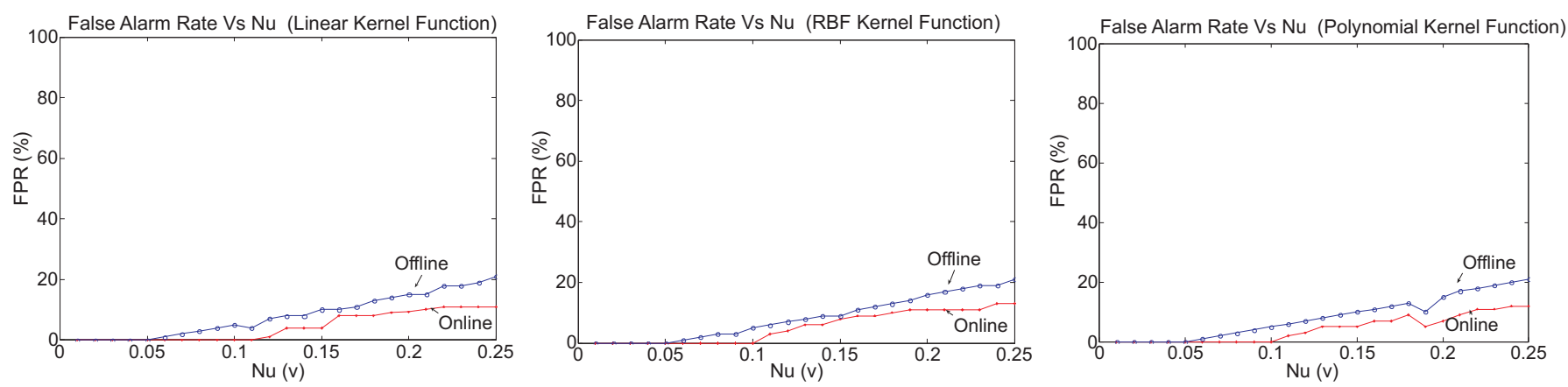

Fig. 5. Synthetic data: false alarm rate for linear (left), $\operatorname{RBF}($ middle) and polynomial (right) kernel functions in terms of parameter $(v)$ selection.
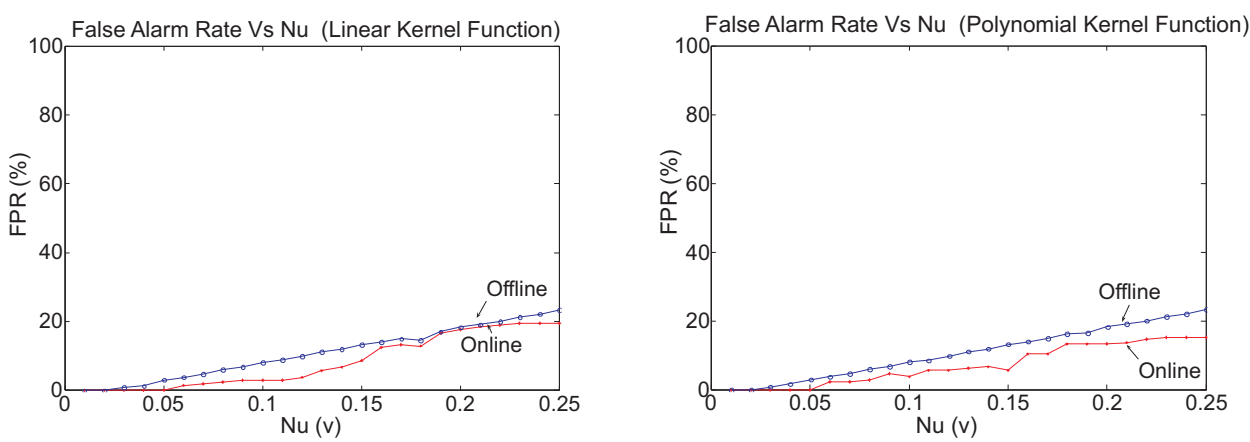

Fig. 6. Real data: false alarm rate for linear (left), polynomial (right) kernel functions in terms of parameter $(v)$ selection.

Figure (5) shows the false alarm rate obtained for the two techniques using the linear, RBF and polynomial kernel functions for the synthetic data. Figure (6) shows the false alarm rate obtained for the two techniques using the linear and polynomial kernel functions for the real data. The simulation results show that our technique achieves lower false alarm rate in terms of parameter selection using different kernel functions compared to the offline outlier detection technique of [5].

\section{CONCLUSIONS}

In this paper, we have proposed an online and local outlier detection technique with low resource consumption based on an unsupervised centered quarter-sphere SVM. We compare the performance of our approach with an offline scheme using synthetic and real data of the Intel Berkeley Research Laboratory. Experimental results show that our approach achieves better mining performance in terms of parameter selection with different kernel functions. We have also presented preliminary work on distinction mechanisms between events and errors. Our future research includes online updating the boundary of normal data with arrival of new data measurements, and evaluating outlier detection performance while distinguishing between events and errors. In addition, we further work on using cross-layer information provided by the underlying MAC layer to increase the robustness of the protocol in presence of network topology changes.

\section{ACKNOWLEDGMENT}

This work is supported by the EU's Seventh Framework Programme and the SENSEI project.

\section{REFERENCES}

[1] I. F. Akyildiz, W. Su, Y. Sankarasubramaniam ,and E. Cayirci, Wireless Sensor Networks: A Survey, Computer Networks, vol. 38, no. 4, pp. $393-$ 422, March 2002.

[2] A. Perrig, J. Stankovic, and D. Wagner, Security in Wireless Sensor Networks, CACM, vol. 47, no. 6, pp. 5357, 2004.

[3] N. R. Prasad and M. Alam, Security Framework for Wireless Sensor Networks, Wireless Personal Communications, vol. 37, no. 3-4, 2006.

[4] V. Chandola, A. Banerjee, and V. Kumar, Outlier Detection: A Survey, Technical Report, University of Minnesota, 2007.

[5] S. Rajasegarar, C. Leckie, M. Palaniswami, and J. C. Bezdek, Quarter Sphere based Distributed Anomaly Detection in Wireless Sensor Networks, in IEEE International Conference on Communications, (Glasgow), pp. 3864-3869, June 2007.

[6] Y. Zhang, N. Meratnia, and P. J. M. Havinga, Outlier Detection Techniques for Wireless Sensor Network: A Survey, Technical Report, University of Twente, 2008.

[7] B. Scholkopf, J. C. Platt, J. C. Shawe-Taylor, A. J. Smola, and R. C Williamson, Estimating the Support of a High-Dimensional Distribution, Neural Computation., vol. 13, no. 7, pp. 14431471, 2001.

[8] D. M. J. Tax and R. P. W. Duin, Support Vector Data Description, Machine Learning, vol. 54, no. 1, pp. 4566, 2004.

[9] P. Laskov, C. Schafer, and I. Kotenko, Intrusion Detection in Unlabeled Data with Ouarter Sphere Support Vector Machines, in Detection of Intrusions and Malware \& Vulnerability Assessment, (Dortmund), 2004.

[10] P. Buonadonna, D. Gay, J. M. Hellerstein, W. Hong, and S. Madden, Task: Sensor Network in a Box, in proceeding of second european workshop on wireless sensor networks, pp. 133-144, 2005.

[11] http://db.csail.mit.edu/labdata/labdata.html

[12] B. Krishnamachari and S. Iyengar, Distributed Bayesian Algorithms for Fault-Tolerant Event Region Detection in Wireless Sensor Networks, IEEE Transactions on Computers, vol. 53, no. 3, pp. 241- 250, 2004.

[13] P. N. Tan, M. Steinback, and V. Kumar, Introduction to Data Mining, Addison Wesley, 2006.

[14] S. G. Nash and A. Sofer, Linear and Nonlinear Programming. McGrawHill, 1996. 